\title{
Late Triassic (Early to Middle Norian) radiolarians from the Antalya Nappes, Antalya, SW Turkey
}

\author{
UGUR KAGAN TEKIN ${ }^{1} \&$ TUGRUL SUKRU YURTSEVER ${ }^{2}$ \\ ${ }^{1}$ Hacettepe Üniversitesi, Jeoloji Mühendisligi Bölümü, 06532 Beytepe, Ankara, Turkey (e-mail: uktekin@yahoo.com). \\ ${ }^{2}$ Maden Tetkik ve Arama Genel Müdürlügü, Jeoloji Etüdleri Dairesi, 06520 Balgat, Ankara, Turkey (e-mail: tsyurtsever@yahoo.com).
}

\begin{abstract}
The Gökdere Formation of the Alakircay Nappe (Antalya Nappes) mainly consists of cherty limestone and limestone with calciturbidite intercalations. Moderately to well-preserved radiolarians were obtained from the Gökcam section of the Gökdere Formation located to the west of Antalya City, southwestern Turkey. The radiolarians of the Gökdere Formation obtained in this study are comparable to the faunas described from the Queen Charlotte Islands, British Columbia and the Antalya Nappes, southwestern Turkey. The age range of the radiolarians is late Early Norian to early Middle Norian based on the co-occurrence of Capnodoce serisa, Harsa siscwaiensis, Xiphosphaera fistulata and an associated fauna. Three species (Nodocapnuchosphaera altineri, Renzium whalenae and Enoplocampe(?) norica) and one subspecies (Kinyrosphaera helicata goekcamensis) are defined as new. J. Micropalaeontol. 22(2): 147-162, November 2003.
\end{abstract}

\section{INTRODUCTION}

The Antalya Nappes, in the Taurides of southern Turkey, include widely exposed Mesozoic radiolaria-bearing pelagic sediments. The middle (Alakircay) and upper (Tahtalidag) nappes of the Antalya Nappes are very important targets for detailed radiolarian biostratigraphic studies. The radiolarian biostratigraphy of the Gökdere Formation (Alakircay Nappe, Antalya Nappes) has been investigated by various workers. Early Norian radiolarians from this formation in the Ispartacay section (near Isparta City, western Taurides) have been reported by De Wever et al. (1979) and De Wever (1982). Detailed biostratigraphical work on the latest Carnian-earliest Norian to Early Norian radiolarians from the Gökdere Formation of the Gürleyikdere section (near Kemer Town, Antalya, western Taurides) have been undertaken by Tekin (1999). Tekin (2002a) recently documented the Late Norian-Rhaetian radiolarians from the Hocaköy section (near Akseki Town, Antalya, central Taurides).

The aim of the present study is to clarify the late Early to early Middle Norian radiolarians from the Gökdere Formation of the Gökcam section and correlate them with the previously described Tethyan and Circum-Pacific faunas.

\section{GEOLOGICAL SETTING}

The Taurides, one of the major components of the AlpineHimalayan Orogenic belt, are located in the southern part of Turkey. They are composed of allochthonous and autochthonous units with a wide range of lithologies. Allochthonous units were assigned to 'nappes' by Brunn et al. (1971), whereas Özgül $(1976,1984)$ adopted the term 'tectonostratigraphic units' for both the allochthonous and autochthonous sequences.

The Antalya Nappes, as a part of the Taurides, were first described and named as a single nappe (Antalya Nappe) in the Antalya region by Lefevre (1967). Brunn et al. (1971) attempted to subdivide it into three slices; the 'Cataltepe Unit' (lower nappe), the 'Alakircay Unit' (middle nappe), and the 'Tahtalidag Unit' (upper nappe). Recently, Senel et al. (1992) have subdivided the Antalya Nappes into four units; the 'Cataltepe Nappe', the 'Alakircay Nappe', the 'Tahtalidag Nappe' and the 'Tekirova Ophiolitic Nappe'.

The Alakircay Nappe is extensively exposed between the Antalya Gulf and the Beydaglari Mountains, and is composed mainly of Triassic to Cretaceous pelagic sediments (Figs $1 \& 2$ ). In the study area, the Triassic rock units of the Alakircay Nappe include the Tesbihli, Candir and Gökdere formations (Senel, 1997a; Fig. 3A). These formations were first named and described by Kalafatcioglu (1973).

The Tesbihli Formation is composed of alternating thin- to medium-bedded, red to reddish brown cherts and shales. The age of the formation is reported to be Late Ladinian to Early Carnian (Senel, 1997a; Tekin, 1999). The Candir Formation consists of thin- to thick-bedded, grey-green, green and yellowish-green, plant-bearing sandstones, shales and claystones. Locally, basalt, conglomerate, limestone, and sandyclayey limestone lenses occur as minor components. The Tesbihli Formation varies in age from Late Anisian to Norian in the region (Senel, 1997a).

The Gökdere Formation includes thin- to medium-bedded, beige-grey limestone and cherty limestone. It occasionally contains calcarenites, shales, bedded cherts, tuffs and pillow lavas. The Gökdere Formation varies in age from Carnian to Rhaetian (Tekin, 1999, 2002a).

In the study area, the Ballik and Kecili Formations are found in the Jurassic-Cretaceous part of the Alakircay Nappe (Senel, 1997a; Fig. 3A). The Ballik Formation was first described by Robertson \& Woodcock (1981) and is characterized by thin- to medium-bedded, red, reddish-brown, green, grey and dark grey chert and shale interbeds with some limestone intercalations. Its age can be assigned to the Early Jurassic to Middle Cretaceous based on radiolaria obtained from the central Taurides (Hocaköy Radiolarite, equivalent of Ballik Formation; Tekin, 2002b). The Upper Cretaceous Kecili Formation was first named by Senel et al. (1981). It is represented by red, pink, green and grey limestones, cherty limestone, chert, shale and calciturbidite in the basal part and olistostromal unit in the upper levels (Senel, 1997a). 


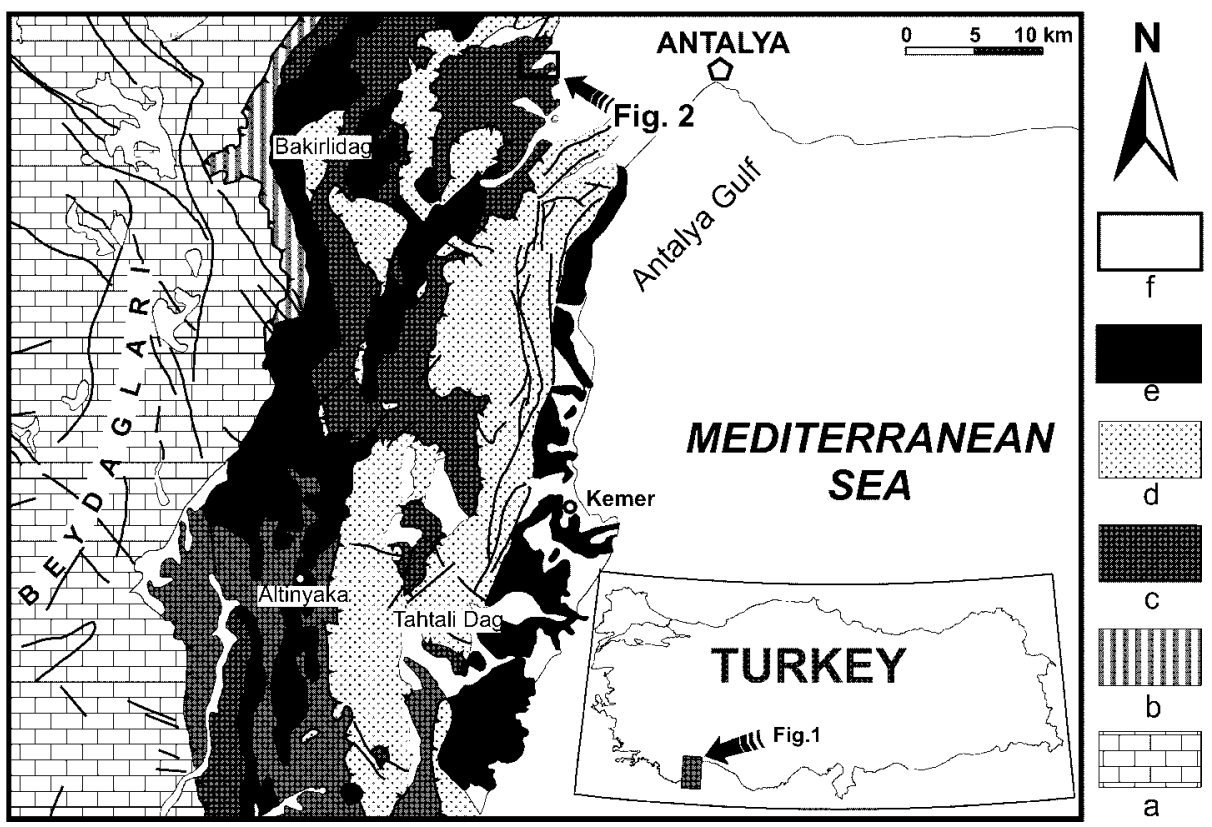

Fig. 1. Major tectonic units of the western part of the Antalya Gulf. Key: a, Beydaglari Autochthonous Unit; b-e, Antalya Nappes - b, Cataltepe Nappe; c, Alakircay Nappe; d, Tahtalidag Nappe; e, Tekirova Ophiolite Nappe including ophiolitic melange; f, post-Lower Miocene units (revised after Senel, 1997a, b).

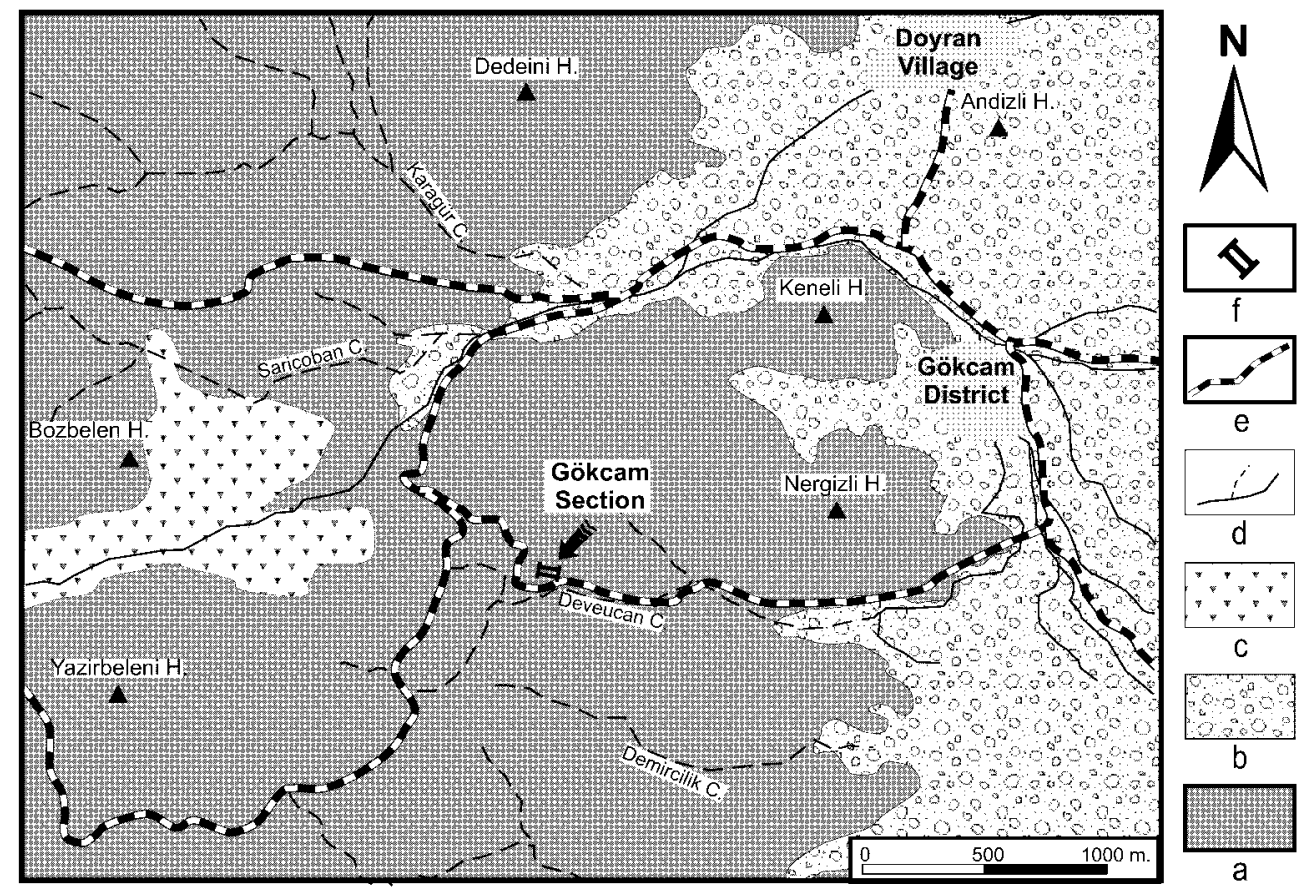

Fig. 2. Geological map of the Gökcam section and its surroundings. Key: a, undifferentiated Alakircay Nappe of the Antalya Nappes; b, Quaternary deposits; c, Quaternary slope debris; d, drainage systems; e, main roads; f, location of the section (revised after Senel, 1997b).

\section{LITHOSTRATIGRAPHY OF THE GÖKCAM SECTION}

The Gökcam section was measured and sampled through the Gökdere Formation of the Alakircay Nappe to the west of Antalya City (Fig. 1). It is situated in the O25a1 quadrangle approximately $2 \mathrm{~km}$ southwest of Gökcam District (Start Point: 40.85.250N, 2.79.500E; End Point: 40.85.275N,
2.79.400E) (Fig. 2). This section is named after the Gökcam District of Doyran village and is located on the northeast bank of the Deveucan Creek (Fig. 2). The Antalya Nappes are represented by several tectonic slices within the study area. The Gökcam section is measured from one of the slices that form the Gökdere Formation. The Gökdere Formation at this location is 


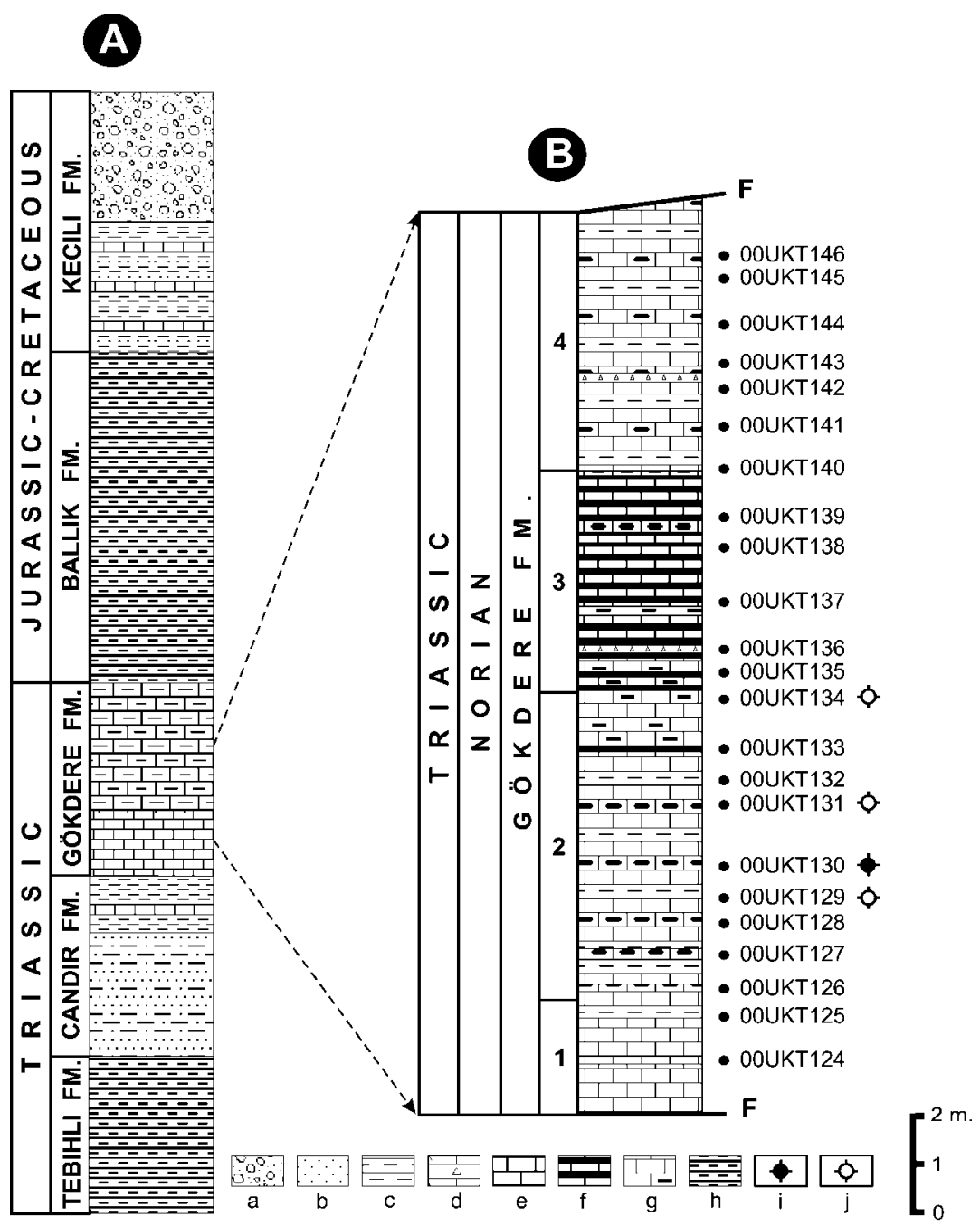

Fig. 3. (A) Generalized columnar section of the Alakircay Nappe of the Antalya Nappes in the study area (not to scale). (B) Gökcam section. Key: a, breccia and conglomerate; b, sandstone; $c$, shale; d, calciturbidite; e, limestone; f, limestone and chert alternations; g, limestone with chert nodules; $\mathrm{h}$, chert and mudstone alternations; i, pyritized Radiolaria; j, normal Radiolaria.

$18.50 \mathrm{~m}$. thick and dominantly characterized by limestones. This section is subdivided into the following four units, from bottom to top (Fig. 3B):

- Unit 1, Limestone without Chert: the lowermost part of the section is mainly characterized by thin- to medium-, occasionally thick-bedded, yellowish-grey to dark grey limestone lacking chert.

- Unit 2, Cherty Limestone and Shale Alternation: limestones in this unit are thin to medium bedded and grey-beige to brownish beige in colour. Grey chert nodules are common in these limestones. Shale beds are very thin and mainly brownyellow in colour. Well-preserved pyritized (sample 00UKT130) and moderately preserved normal late Early to early Middle Norian radiolarians (sample 00UKT129, 00UKT131 and 00UKT134) were obtained from the limestone beds of this unit.

- Unit 3, Chert and Limestone Alternation: the dominant lithologies in this unit are chert and limestone alternations with rare shale and calciturbidite intercalations. The limestones in this part of the succession are again thin to medium bedded, grey to beige in colour, while the cherts are thin bedded and dark grey in colour.

- Unit 4, Cherty Limestone and Shale Alternation with Calciturbidite Intercalations: this uppermost unit is similar to unit two. It is characterized by mainly thin- to medium-, occasionally thick-bedded, grey to beige-coloured limestone with dark grey chert nodules and thin-bedded green, greenish-grey shale interbeds. Rare thin-bedded, grey to beige calciturbidite intercalations are also encountered in this part of the succession.

\section{MATERIALS, METHODS AND REPOSITORY}

Twenty-three samples were collected both from chert nodules/ bands and limestone beds of the Gökdere Formation in the Gökcam section. No radiolarians were obtained from the cherts. Moderately to well-preserved late Early to early Middle Norian radiolarians were extracted from four samples taken from the 
U. K. Tekin \& T. S. Yurtsever

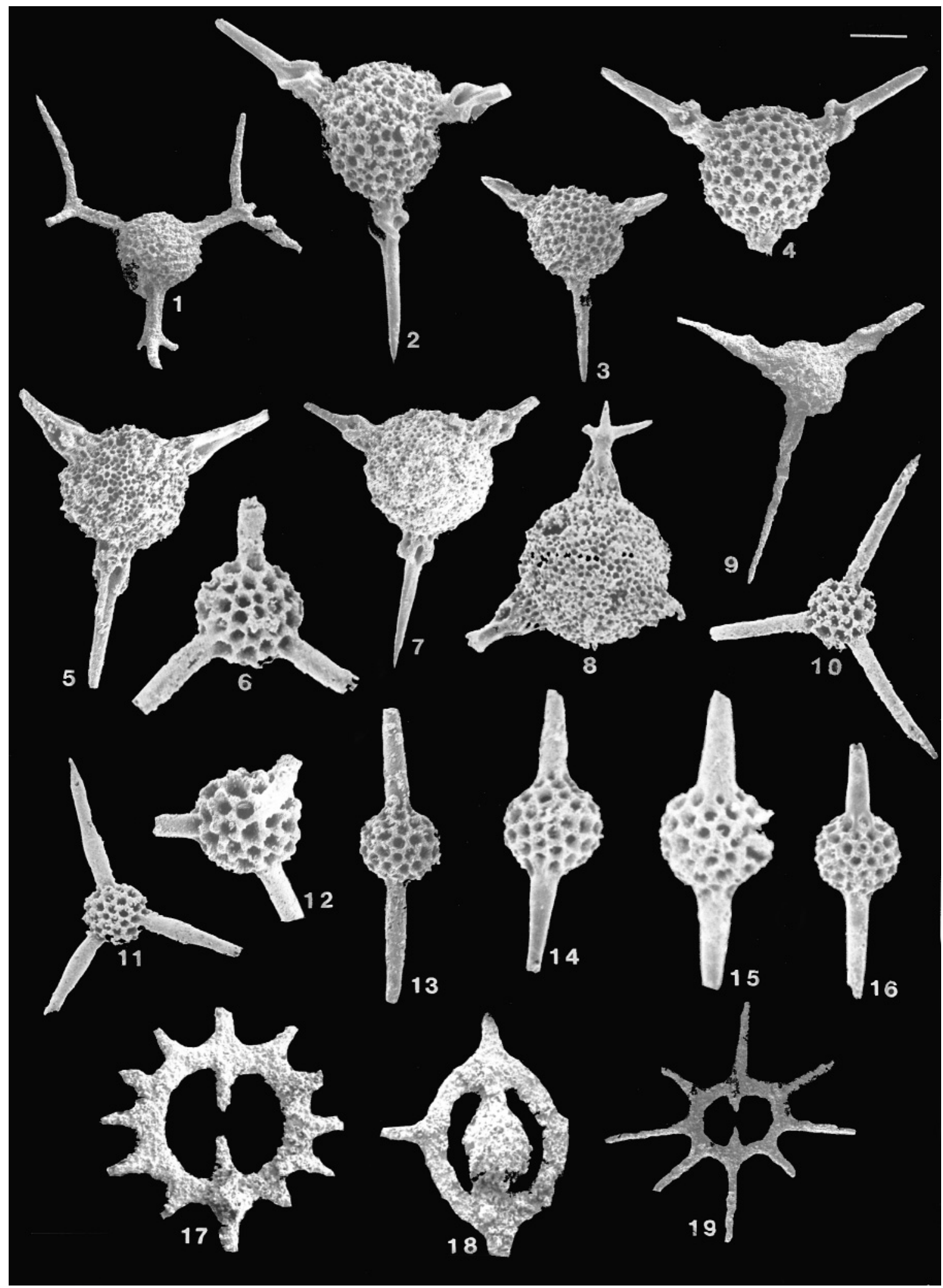


limestone beds. Well-preserved pyritized radiolarians were found only in sample 00UKT130 (Fig. 3B).

While the limestone samples were processed with formic acid (5-10\%), chert samples were etched with diluted hydrofluoric acid (5-10\%) following the Pessagno \& Newport (1972) method. The extracted radiolarians were studied with a stereoscopic microscope. The SEM microscope Zeiss DSM 940A in Innsbruck University, Austria was utilized for the precise identification and illustration of the radiolarians.

All holotypes and paratypes are deposited in the collection of the Natural History Museum, General Directorate of Mineral Research and Exploration, Ankara, Turkey.

\section{SYSTEMATIC DESCRIPTIONS}

In this section the following abbreviations are utilized for the measurements: HT, holotype; Min., minimum; Max., maximum; Av., average; Incl., including.

\section{Phylum Protozoa}

Subclass Radiolaria Müller, 1858

Order Polycystina Ehrenberg 1875

Suborder Spumellaria Ehrenberg, 1838

Family Stylosphaeridae Haeckel, 1882 emend.

Kozur \& Mostler, 1979

Genus Kahlerosphaera Kozur \& Mostler, 1979

Type species. Kahlerosphaera parvispinosa Kozur \& Mostler, 1979.

\section{Kahlerosphaera norica Kozur \& Mock, 1981}

(P1. 1, fig. 1)

1981 Kahlerosphaera norica Kozur \& Mock in Kozur \& Mostler: 36, pl. 15, fig. 4.

1997 Kahlerosphaera norica Kozur \& Mock Group; Sugiyama: 181.

1999 Kahlerosphaera norica Kozur \& Mock Group; Tekin: 65-66, pl. 1, figs 11-12.

Stratigraphic range. Late Triassic; latest Carnian/earliest Norian-early Middle Norian.

Occurrence. Western Carpathians; Mino Terrane, Central Japan; Yaylakuzdere and Gökcam sections, Antalya, southwest Turkey.
Family Capnuchosphaeridae De Wever, 1979 emend. Pessagno, 1979 emend. Blome, 1983

Subfamily Capnuchosphaerinae De Wever, 1982 Genus Kinyrosphaera Bragin, 1999

Type species. Kinyrosphaera trispinosa Bragin, 1999.

Kinyrosphaera helicata Bragin, 1999

Kinyrosphaera helicata goekcamensis Tekin n. subsp.

(Pl. 1, figs 2-4)

Derivation of name. This species is named after the type locality, Gökcam District, Doyran village, Antalya City.

Diagnosis. Capnuchosphaerid with spherical, double-layered cortical shell. Outer layer of cortical shell consisting of large, polygonal pore frames with highly elevated nodes at pore frame vertices. Inner pore frames small and mainly not preserved. Three tumidispinae are situated in the same plane. Spinal tunnels of tumidispinae short, wide and strongly sinistrially twisted at distal end. Spinal shafts long and needle-like.

Holotype. From the sample 00UKT130, illustrated in Plate 1, figure 2.

Material. Nine specimens.

Type locality. Gökcam section, Antalya, southwest Turkey (see locality descriptions).

Horizon. Upper Triassic; upper Lower Norian-lower Middle Norian.

Description. Cortical shell, spherical with double-layered shell structure. Outer layer of meshwork consisting of large, polygonal (mainly hexagonal) pore frames with highly elevated nodes at pore frame vertices. Inner pore frames small, polygonal with circular to sub-circular pores. Inner pore frames occasionally observed. Three tumidispinae are situated in the same plane. Spinal tunnels of tumidispinae short, wide, sub-cylindrical with small to medium-sized pores arranged in longitudinal rows. Distal part of the spinal tunnels strongly twisted. Spinal tumours prominent. Spinal shaft, long, needle-like, circular in axial section. Usually one of the spinal shafts longer than the other spinal shafts.

Explanation of Plate 1. Scanning electron photomicrographs of late Early to early Middle Norian Spumellaria from the Gökdere Formation of the Gökcam section. Length of scale bar is number of micrometres $(\mu \mathrm{m})$ for each figure. fig. 1. Kahlerosphaera norica Kozur \& Mock, sample 00UKT130; scale bar $140 \mu \mathrm{m}$. figs 2-4. Kinyrosphaera helicata goekcamensis n. subsp.: 2, holotype, sample 00UKT130, scale bar $100 \mu$ m; 3-4, paratypes, both specimens from sample 00UKT130, scale bar $85 \mu \mathrm{m}$ and $100 \mu \mathrm{m}$, respectively. figs 5, 7. Nodocapnuchosphaera altineri n. sp.: 5, holotype, sample 00UKT130, scale bar $100 \mu \mathrm{m}$; 7, paratype, sample 00UKT130, scale bar $100 \mu \mathrm{m}$. fig. 6. Capnodoce crystallina Pessagno, sample 00UKT130; scale bar $70 \mu \mathrm{m}$. fig. 8. Nodocapnuchosphaera tuzcuae Tekin, sample 00UKT130; scale bar $75 \mu \mathrm{m}$. fig. 9. Sarla dumitricai (Lahm), sample 00UKT129; scale bar $100 \mu \mathrm{m}$. figs 10-11. Capnodoce serisa De Wever, both specimens from sample 00UKT130; scale bar $140 \mu \mathrm{m}$ and $125 \mu \mathrm{m}$, respectively. fig. 12. Loffa sp. A, sample 00UKT130; scale bar $75 \mu \mathrm{m}$. fig. 13. Renzium adversum Blome, sample 00UKT130; scale bar $75 \mu \mathrm{m}$. figs 14-16. Renzium whalenae n. sp.: 14, holotype, sample 00UKT130, scale bar $55 \mu \mathrm{m}$; 15-16, paratypes, both specimens from sample 00UKT130, scale bar $55 \mu \mathrm{m}$ and $65 \mu \mathrm{m}$, respectively. fig. 17. Palaeosaturnalis dumitricai Tekin, sample 00UKT129; scale bar $55 \mu \mathrm{m}$. fig. 18. Palaeosaturnalis latiannulatus Kozur \& Mostler, sample 00UKT129; scale bar $65 \mu \mathrm{m}$. fig. 19. Praehexasaturnalis burnsensis (Blome), sample 00UKT134; scale bar $150 \mu \mathrm{m}$. 
Dimensions $(\mu \mathrm{m})$. Based on six specimens.

$\begin{array}{lrrrr} & \text { HT } & \text { Min. } & \text { Max. } & \text { Av. } \\ & 208 & 178 & 225 & 199 \\ \text { Diameter of the cortical shell } & 50 & 48 & 62 & 53 \\ \text { Width of tumidispinae (proximally) } & 267 & 238 & 275 & 260 \\ \text { Length of tumidispinae (incl. tips) } & & & & \end{array}$

Stratigraphic range. Late Triassic; late Early Norian-early Middle Norian.

Occurrence. Gökcam section, Antalya, southwest Turkey.

Remarks. Kinyrosphaera helicata goekcamensis Tekin n. subsp. differs from $K$. helicata helicata Bragin by having highly elevated outer pore frames with prominent nodes at pore frame vertices, shorter spinal tunnels and long, unequal spinal shafts.

\section{Kinyrosphaera helicata helicata Bragin, 1999}

1995 Capnuchosphaera? sp. Halemic \& Gorican: pl. 2, fig. 10. 1999 Kinyrosphaera helicata Bragin in Bragin \& Krylov: 551, figs $5 \mathrm{~F}, \mathrm{H}, 6 \mathrm{~A}$.

1999 Capnuchosphaera deweveri Kozur \& Mostler: Tekin; 70-71, pl. 3, figs $12-13$.

Stratigraphic range. Late Triassic; Early Norian-early Middle Norian.

Occurrence. Croatia; Agia Varvara Village, Mamonia CompIex, Cyprus; Yaylakuzdere section, Antalya, southwest Turkey.

Genus Nodocapnuchosphaera Tekin, 1999 emend. herein

Type species. Nodocapnuchosphaera tuzcuae Tekin, 1999.

Emended definition. Spinal tunnels of tumidispinae mainly very short, porous. Pores on spinal tunnels arranged in longitudinal rows. Spinal shaft may be branched off at its terminations.

Remarks. Nodocapnuchosphaera Tekin differs from Kinyrosphaera Bragin by having uniform, large nodular nodes on cortical shell.

\section{Nodocapnuchosphaera altineri Tekin n. sp.}

(Pl. 1, figs 5, 7)

1998 Acaeniotyle ? sp. A Cordey: 54, pl. 13, fig. 1.

Derivation of name. This species is named after Prof. Dr Demir Altiner, Middle East Technical University, Ankara, Turkey, in honour of his great contribution to the knowledge of Palaeozoic and Mesozoic foraminiferal biostratigraphy.

Diagnosis. Capnuchosphaerid with spherical, double-layered cortical shell. Three tumidispinae situated in same plane. Spinal tunnels very short, porous. Straight spinal tumours ended with spinal shafts. One of the spinal shafts longer than the others.

Holotype. From the sample 00UKT130, illustrated in Plate 1, figure 5 .
Material. 11 specimens.

Type locality. Gökcam section, Antalya, southwest Turkey (see locality descriptions).

Horizon. Upper Triassic; upper Lower Norian-lower Middle Norian.

Description. Cortical shell large, spherical and double layered. Outer layer of meshwork consisting of large, polygonal pore frames. Inner pore frames with mainly hexagonal, sometimes pentagonal, smaller pore frames. Surface of cortical shell undulated related to occurrence of large, uniform nodes. Nodes separated from each other by relatively shallow depressions. Three tumidispinae extend out from cortical shell, situated in the same plane. Spinal tunnels very short, cylindrical with big pores arranged in longitudinal rows. Spinal tumours straight, not twisted. Spinal pores prominent, large, sub-circular. Spinal shaft long, triradiate in proximal part then needle like, circular in cross-section. One spinal shaft usually longer than the other spinal shafts.

Dimensions $(\mu \mathrm{m})$. Based on five specimens.

$\begin{array}{lrrrr} & \text { HT } & \text { Min. } & \text { Max. } & \text { Av. } \\ & 192 & 192 & 200 & 197 \\ \text { Diameter of the cortical shell } & 58 & 50 & 58 & 54 \\ \text { Width of tumidispinae (proximally) } & 233 & 216 & 233 & 223 \\ \text { Maximum length of tumidispinae } & & & & \end{array}$

Stratigraphic range. Late Triassic; late Early Norian-early Middle Norian.

Occurrence. Hozameen Complex, British Columbia; Gökcam section, Antalya, southwest Turkey.

Remarks. Nodocapnuchosphaera altineri Tekin n. sp. differs from $N$. tuzcuae Tekin in having a slightly smaller cortical shell, longer tumidispinae and an absence of branching spinal shafts.

$$
\text { Nodocapnuchosphaera tuzcuae Tekin, } 1999
$$$$
\text { (P1. 1, fig. 8) }
$$

1999 Nodocapnuchosphaera tuzcuae Tekin: 81, pl. 6, figs 10-14.

Stratigraphic range. Late Triassic; latest Carnian/earliest Norian-early Middle Norian.

Occurrence. Yaylakuzdere and Gökcam sections, Antalya, southwest Turkey.

\section{Subfamily Sarlinae De Wever, 1982 \\ Genus Sarla Pessagno, 1979}

Type species. Sarla prietoensis Pessagno, 1979.

Sarla dumitricai (Lahm, 1984)

(Pl. 1, fig. 9)

1984 Triactoma dumitricai Lahm: 73, pl. 13, fig. 1.

pars 1999 Vinassaspongus transitus Kozur \& Mock; Bragin \& 
Triassic radiolaria from SW Turkey

Krylov: 546, fig. 6D non fig. 6F (=Sarla transitus (Kozur \& Mock).

1999 Sarla dumitricai (Lahm); Tekin: 86, pl. 8, figs 6, 10.

Stratigraphic range. Middle Triassic; Early Ladinian-Late Triassic; early Middle Norian.

Occurrence. Recaoro, Italy; Mamonia Complex, Cyprus; Yaylakuzdere and Gökcam sections, Antalya, southwest Turkey.

Family Pantanelliidae Pessagno, 1977 emend. Pessagno \& Blome, 1980

Subfamily Capnodocinae Pessagno, 1979 emend. Blome, 1983

Genus Capnodoce De Wever, 1979 emend Pessagno, 1979

Type species. Capnodoce anapates De Wever, 1979.

Capnodoce crystallina Pessagno, 1979 Group

(Pl. 1, fig. 6)

1979 Capnodoce crystallina Pessagno in Pessagno, Finch \& Abbott: 176, pl. 1, figs 1-3.

1983 Capnodoce antiqua Blome: 24, pl. 5, figs 4, 12, 17.

1984 Capnodoce antiqua Blome; Blome: 33, pl. 4, fig. 6.

1984 Capnodoce crystallina Pessagno; Blome: 34.

1986 Capnodoce antiqua Blome; Yoshida: pl. 10, figs 4, 5.

1986 Capnodoce crystallina Pessagno; Sato, Murata \& Yoshida:

figs 16 (11-12).

1986 Capnodoce antiqua Blome; Bragin: pl. 2, fig. 1.

1991 Capnodoce antiqua Blome; Bragin: 83, pl. 6, fig. 10.

1997 Capnodoce cf. antiqua Blome; Knipper, Satian \& Bragin: pl. 1, fig. 2.

1997 Capnodoce crystallina Pessagno Group; Sugiyama: 175, fig. 49 (17).

1999 Capnodoce crystallina Pessagno Group; Tekin: 92-93, pl. 11, figs 2-3.

Stratigraphic range. Late Triassic; latest Carnian/earliest Norian-early Middle Norian-?late Middle Norian.

Occurrence. Baja California Sur, Mexico; southwest and central Japan; East-Central Oregon, USA; Far east Russia; Sevan-Akera, Lesser Caucasus; Yaylakuzdere and Gökcam sections, Antalya, southwest Turkey.

Capnodoce serisa De Wever, 1979

(Pl. 1, figs 10-11)

pars 1979 Capnodoce serisa De Wever in De Wever, San

Flippo, Riedel \& Gruber: 82, pl. 2, fig. 9 non pl. 2, fig. 8 (=Loffa sp.).

1979 Capnodoce serisa De Wever; Nakaseko \& Nishimura: 75, pl. 6, figs $1,2$.

non 1981 Capnodoce serisa De Wever; Kozur \& Mostler: pl. 63, fig. 2 (=Capnodoce longibrachium Tekin).

1982 Capnodoce serisa De Wever; De Wever: 143-145, pl. 3, figs $1-3$.

1982 Capnodoce serisa De Wever; Yao: pl. 2, fig. 24.
1982 Capnodoce serisa De Wever; Yao, Matsuoka \& Nakatani: pl. 1, fig. 22.

1982 Capnodoce serisa De Wever; Kishida \& Sugano: pl. 2, fig. 6 .

1982 Capnodoce serisa De Wever; Sato, Nishizono \& Murata: pl. 2, fig. 6 .

1983 Capnodoce serisa De Wever; Nishizono \& Murata: pl. 2, fig. 9.

1983 Capnodoce fragilis Blome: 26, pl. 6, figs 4, 10, 18, pl. 11, fig. 5.

1984 Capnodoce fragilis Blome; Blome: 34, pl. 4, fig. 11.

1986 Capnodoce serisa De Wever; Sato, Murata \& Yoshida: fig. 16 (10).

1986 Capnodoce fragilis Blome; Yoshida: pl. 10, fig. 6.

1989 Capnodoce fragilis Blome; Carter, Orchard \& Tozer: pl. 1, fig. 10 .

1991 Capnodoce fragilis Blome; Carter: 199, pl. 1, figs 1, 6.

1996 Capnodoce fragilis Blome; Yeh \& Cheng: pl. 10, figs $1,5$.

1997 Capnodoce serisa De Wever; Sugiyama: 175, fig. 49 (16).

1999 Capnodoce serisa De Wever; Tekin: 94-95, pl. 11, figs 12-13.

Stratigraphic range. Late Triassic; Early Norian-Late Norian.

Occurrence. Sicily, Italy; Ispartacay, Isparta and Yaylakuzdere and Gökcam sections, Antalya, southwest Turkey; southwest and central Japan; East-Central Oregon, USA; Queen Charlotte Islands, British Columbia, Canada; Busuanga Island, Philippines.

Genus Loffa Pessagno, 1979

Type species. Loffa mulleri Pessagno, 1979.

Loffa sp. A

(Pl. 1, fig. 12)

Description. Cortical shell with coarse pentagonal and hexagonal pore frames with massive nodes at vertices. Primary spines short, tubular, straight and maintaining approximately same diameter over all length.

Stratigraphic range. Late Triassic; late Early Norian-early Middle Norian.

Occurrence. Gökcam section, Antalya, southwest Turkey.

Remarks. Loffa sp. A in this study differs from $L$. vesterensis Blome in having a spherical cortical shell and shorter primary spines that are the same diameter over all their length.

Genus Renzium Blome, 1983

Type species. Renzium webergorum Blome, 1983.

Renzium adversum Blome, 1983

(P1. 1, fig. 13)

1983 Renzium adversum Blome: 40, 42, pl. 10, figs 1, 6, 7, 12.

1984 Renzium adversum Blome; Blome: 36, pl. 5, fig. 2.

1999 Renzium adversum Blome; Tekin: 96, pl. 12, fig. 5. 
Stratigraphic range. Late Triassic; Early Norian-early Middle Norian-?late Middle Norian.

Occurrence. East-Central Oregon, USA; Yaylakuzdere and Gökcam sections, Antalya, southwest Turkey.

Renzium whalenae Tekin n. sp.

(Pl. 1, figs 14-16)

Derivation of name. This species is named after Dr Patricia A. Whalen, University of Arkansas, USA, in honour of her contribution to the study of Liassic radiolarian biostratigraphy.

Diagnosis. A species of Renzium with a spherical to sub-spherical cortical shell. Meshwork consisting of pentagonal and hexagonal pore frames. Primary spines moderately long, tubular, unequal, tapering distally.

Holotype. From the sample 00UKT130, illustrated in Plate 1, figure 14 .

Material. Eight specimens.

Type locality. Gökcam section, Antalya, southwest Turkey (see locality descriptions).

Horizon. Upper Triassic; upper Lower Norian-lower Middle Norian.

Description. Cortical shell small, spherical to sub-spherical in outline. Meshwork consists of a mixture of pentagonal and hexagonal (predominantly hexagonal) pore frames. Pore frame vertices with very short, rounded nodes. Bars of pore frames thinner along $\mathrm{Y}$ than $\mathrm{Z}$. Six to seven pores visible on cortical shell along an axis in line with polar spines. Primary spines moderately long, tubular, unequal and tapering distally. No triradiate distal portion visible.

Dimensions $(\mu \mathrm{m})$. Based on five specimens.

Diameter of the cortical shell

HT Min. Max. Av. $\begin{array}{lllll}\text { (perpendicular to main spines) } & 93 & 91 & 100 & 95\end{array}$

$\begin{array}{lllll}\text { Length of short primary spines } & 80 & 80 & 83 & 81\end{array}$

$\begin{array}{lllll}\text { Length of long primary spines } & 93 & 86 & 118 & 102\end{array}$

$\begin{array}{lllll}\text { Max. width of primary spines } & 20 & 20 & 27 & 24\end{array}$

Stratigraphic range. Late Triassic; late Early Norian-early Middle Norian.

Occurrence. Gökcam section, Antalya, southwest Turkey.

Remarks. Renzium whalenae Tekin n. sp. differs from Renzium adversum Blome in having unequal, shorter and tapering main spines.

Family Acanthocircidae Pessagno, 1977

Genus Palaeosaturnalis Donofrio \& Mostler, 1978 emend. Kozur \& Mostler, 1983
Type species. Spongosaturnalis triassicus Kozur \& Mostler, 1972. Palaeosaturnalis dumitricai Tekin, 1999

(P1. 1, fig. 17)

1991 Palaeosaturnalis karnicus (Kozur \& Mostler); Bragin: 92, pl. 6, fig. 11.

1999 Palaeosaturnalis dumitricai Tekin: 109, pl. 16, figs 4-6.

Stratigraphic range. Late Triassic; latest Carnian/earliest Norian-early Middle Norian.

Occurrence. Far East Russia; Yaylakuzdere and Gökcam sections, Antalya, southwest Turkey.

Palaeosaturnalis latiannulatus Kozur \& Mostler, 1983

(Pl. 1, fig. 18)

1983 Palaeosaturnalis latiannulatus Kozur \& Mostler: 20, pl. 5, fig. 1.

1999 Palaeosaturnalis latiannulatus Kozur \& Mostler; Bragin \& Krylov: 556, fig. 9D.

1999 Palaeosaturnalis latiannulatus Kozur \& Mostler; Tekin: 110, pl. 11, figs 1-2.

Stratigraphic range. Late Triassic; latest Carnian/earliest Norian-early Middle Norian.

Occurrence. Western Carpathians; Agia Varvara Village, Mamonia Complex, Cyprus; Yaylakuzdere and Gökcam sections, Antalya, southwest Turkey.

Genus Praehexasaturnalis Kozur \& Mostler, 1983

Type species. Palaeosaturnalis tenuispinosus Donofrio \& Mostler, 1978.

Praehexasaturnalis burnsensis (Blome, 1984)

(Pl. 1, fig. 19)

1984 Acanthocircus burnsensis Blome: 21-22, pl. 1, figs 1, 11 1984 Acanthocircus lupheri Blome: 23-24, pl. 1, figs 10, 18. 1984 Acanthocircus macoyensis Blome: 24, pl. 2, figs 1, 12. 1984 Acanthocircus ochocoensis Blome: 24, pl. 2, figs 2, 13. 1984 Acanthocircus prinevillensis Blome: 24, pl. 2, figs 3, 14. 1990 Praehexasaturnalis burnsensis (Blome); Kozur \& Mostler: 194. 1991 Palaeosaturnalis prinevillensis (Blome); Yang \& Mizutani: 66-67, pl. 2, figs 7, 8; pl. 3, figs 3, 5, 9.

1998 Palaeosaturnalis prinevillensis (Blome); Carter, Whalen \& Guex: 54, pl. 14, figs ?19, 23.

1999 Praehexasaturnalis burnsensis (Blome); Tekin: 111-112, pl. 18, fig. 1.

Stratigraphic range. Late Triassic; late Early Norian-Early Jurassic; Sinemurian.

Occurrence. East-Central Oregon, USA; northeast China; Queen Charlotte Islands, British Columbia, Canada; Yaylakuzdere and Gökcam sections, Antalya, southwest Turkey.

Praehexasaturnalis tenuispinosus (Donofrio \& Mostler, 1978) (P1. 2, fig. 1) 


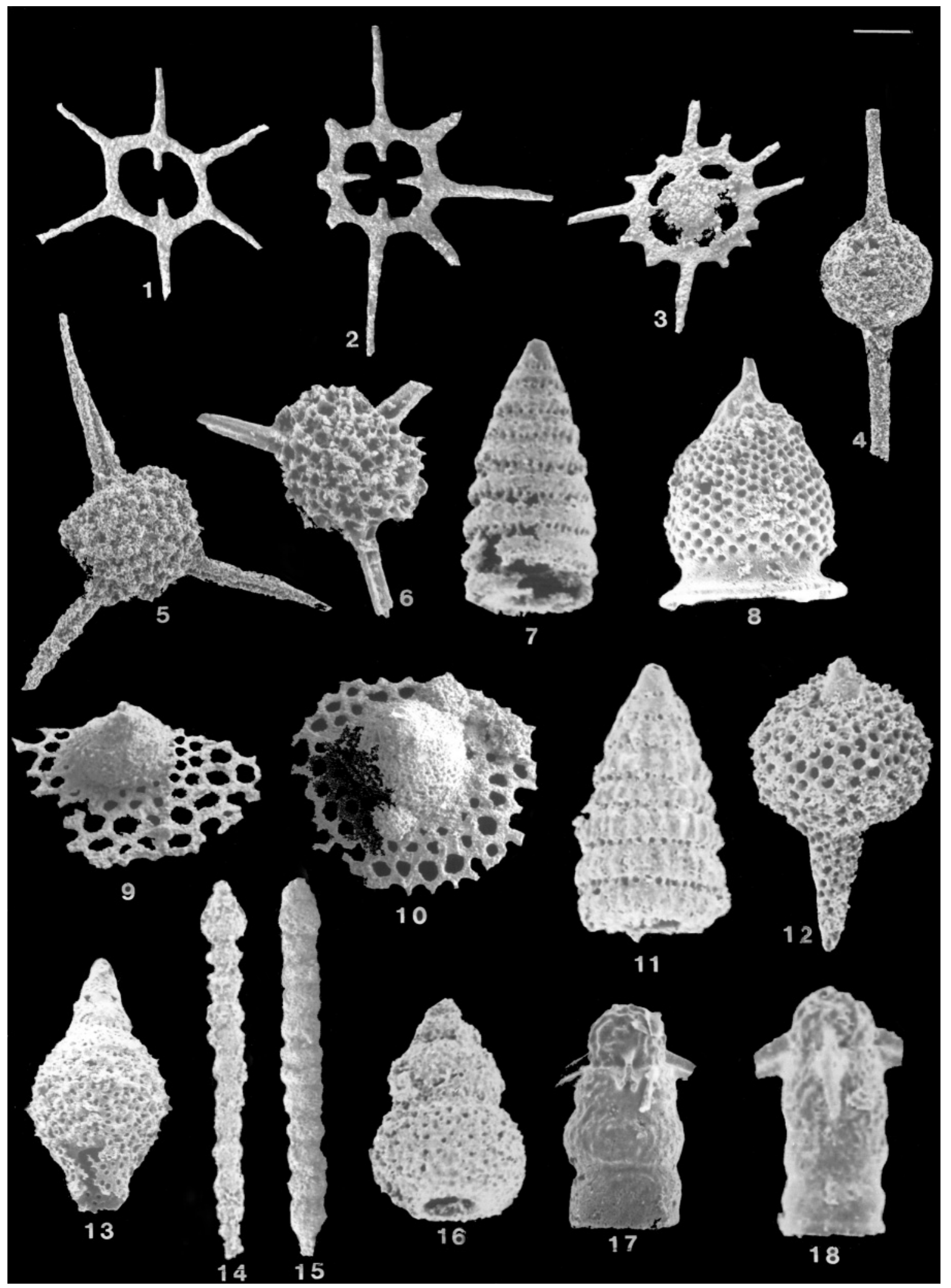


1978 Palaeosaturnalis tenuispinosus Donofrio \& Mostler: 37-38, pl. 7, figs $1-3,8$.

1979 Spongosaturnalis sp. cf. S. elegans Kozur \& Mostler; De Wever, San Flippo, Riedel \& Gruber: 81, pl. 2, figs 3, 4 .

1982 Palaeosaturnalis aff. tenuispinosus Donofrio \& Mostler; Yao: pl. 3, fig. 17.

1982 Acanthocircus tenuispinosus (Donofrio \& Mostler);

De Wever: 206-207, pl. 13, figs 3-5.

1983 Praehexasaturnalis tenuispinosus (Donofrio \& Mostler); Kozur \& Mostler: 30.

1984 Acanthocircus vigrassi Blome: 26, pl. 2, figs 9-10.

1986 Acanthocircus cf. A. elegance Kozur \& Mostler; Yoshida:

pl. 15 , figs 8,9 .

1990 Acanthocircus tenuispinosus (Donofrio \& Mostler); Yeh: 17, pl. 13, fig. 3.

1990 Praehexasaturnalis tenuispinosus (Donofrio \& Mostler); Kozur \& Mostler: 194.

1997 Praehexasaturnalis tenuispinosus (Donofrio \& Mostler); Sugiyama: 185, figs. $51(9,10)$.

1999 Praehexasaturnalis tenuispinosus (Donofrio \& Mostler);

Tekin: 112, pl. 18, fig. 2.

Stratigraphic range. Late Triassic; late Early Norian-Early Jurassic; Hettangian.

Occurrence. Poetschen, Austria; Ispartacay Formation, Isparta and Yaylakuzdere and Gökcam sections, Antalya, southwest Turkey; East-Central Oregon, USA; Inuyama Area, Gifu Prefecture and Mino Terrane Central Japan; Busuanga Island, Philippines.

Genus Stauroacanthocircus Kozur \& Mostler, 1983 emend. Kozur \& Mostler, 1990

Type species. Pseudoheliodiscus concordis De Wever, 1981.

Stauroacanthocircus dickinsoni (Yeh, 1989) n. comb.

(P1. 2, fig. 2)

1989 Quadrisaturnalis dickinsoni Yeh: 50, pl. 13, fig. 18.

Stratigraphic range. Late Triassic; late Early Norian-early Middle Norian.

Occurrence. East-Central Oregon, USA; Gökcam section, Antalya, southwest Turkey.
Remarks. Quadrisaturnalis Yeh, 1989, with four polar spines in cross-section is a junior synonym of Stauroacanthocircus Kozur \& Mostler, 1983.

Stauroacanthocircus ?poetschensis Kozur \& Mostler, 1990 (Pl. 2, fig. 3)

1990 Stauroacanthocircus ?poetschensis Kozur \& Mostler: 199200, pl 7, fig. 8, pl. 8, figs 1, 4 .

1999 Stauroacanthocircus ?poetschensis Kozur \& Mostler; Tekin: 116, pl. 19, figs 7-8.

Stratigraphic range. Late Triassic; Early Norian-Middle Norian.

Occurrence. Pötschenwand, Austria; Yaylakuzdere and Gökcam sections, Antalya, southwest Turkey.

Spumellariina Incertae Sedis

Genus Harsa Carter, 1991

Type species. Harsa siscwaiensis Carter, 1991.

Harsa siscwaiensis Carter, 1991

(Pl. 2, fig. 5)

1991 Harsa siscwaiensis Carter: 199-200, pl. 1, fig. 2.

Stratigraphic range. Late Triassic; late Early Norian-early Middle Norian.

Occurrence. Queen Charlotte Islands, British Columbia; Gökcam section, Antalya, southwest Turkey.

Harsa sp. aff. H. siscwaiensis Carter, 1991

(P1. 2, fig. 6)

1991 Harsa sp. aff. H. siscwaiensis Carter: 200, pl. 1, fig. 3.

Stratigraphic range. Late Triassic; late Early Norian-early Middle Norian.

Occurrence. Queen Charlotte Islands, British Columbia; Gökcam section, Antalya, southwest Turkey.

Genus Xiphosphaera Haeckel, 1882

Type species. Xiphosphaera tredicomporata Rüst, 1885.

Explanation of Plate 2. Scanning electron photomicrographs of late Early to early Middle Norian Spumellaria and Nassellaria from the Gökdere Formation of the Gökcam section. Length of scale bar is number of micrometres $(\mu \mathrm{m})$ for each figure. fig. 1. Praehexasaturnalis tenuispinosus (Donofrio \& Mostler), sample 00UKT134; scale bar $120 \mu \mathrm{m}$. fig. 2. Stauroacanthocircus dickinsoni (Yeh), sample 00UKT134; scale bar 130 $\mu$ m. fig. 3. Stauroacanthocircus ? poetschensis Kozur \& Mostler, sample 00UKT134; scale bar $130 \mu \mathrm{m}$. fig. 4. Xiphosphaera fistulata Carter, sample 00UKT129; scale bar $65 \mu \mathrm{m}$. fig. 5. Harsa siscwaiensis Carter, sample 00UKT130; scale bar $100 \mu \mathrm{m}$. fig. 6. Harsa sp. aff. H. siscwaiensis Carter, sample 00UKT130; scale bar $75 \mu \mathrm{m}$. fig. 7. Japonocampe nova (Yao), sample 00UKT130; scale bar 75 um. fig. 8. Deflandrecyrtium parvus Tekin, sample 00UKT130; scale bar $50 \mu \mathrm{m}$. figs 9-10. Haeckelicyrtium sp. A: 9, sample 00UKT134, scale bar 120 $\mu$ m; 10, sample 00UKT129, scale bar $100 \mu \mathrm{m}$. fig. 11. Whalenella regia (Blome), sample 00UKT130; scale bar $75 \mu \mathrm{m}$. fig. 12. Syringocapsa sp. aff. $S$. batodes De Wever, sample 00UKT130; scale bar $75 \mu \mathrm{m}$. fig. 13. Syringocapsa sp. A, sample 00UKT130; scale bar $50 \mu \mathrm{m}$. fig. 14. Xiphotheca longa Kozur \& Mock, sample 00UKT130; scale bar $100 \mu \mathrm{m}$. fig. 15. Xiphotheca pseudolonga Tekin, sample 00UKT134; scale bar $110 \mu \mathrm{m}$. fig. 16. Canesium lentum Blome, sample 00UKT130; scale bar $55 \mu \mathrm{m}$. figs 17-18. Enoplocampe(?) norica n. sp.: 17, holotype, sample 00UKT130, scale bar 45 $\mu \mathrm{m}$; 18, paratype, sample 00UKT130, scale bar $45 \mu \mathrm{m}$. 
Triassic radiolaria from SW Turkey

Xiphosphaera fistulata Carter, 1991

(Pl. 2, fig. 4)

1991 Xiphosphaera fistulata Carter: 200, pl. 1, figs 4, 5, 7, 8, 9, 10. ?1996 Unnamed Spumellaria Yeh \& Cheng: pl. 11, figs 11, 16. 1997 Xiphosphaera fistulata Carter; Sugiyama: 188, fig. 50 (27). 1999 Xiphosphaera fistulata Carter; Tekin: 123, pl. 23, figs 4-6.

Stratigraphic range. Late Triassic; late Early Norian-early Middle Norian.

Occurrence. Queen Charlotte Islands, British Columbia, Canada; ?Busuanga Island, Philippines; Mino Terrane, Central Japan; Yaylakuzdere and Gökcam sections, Antalya, southwest Turkey.

\section{Suborder Nassellaria Ehrenberg, 1875 \\ Family Canoptidae Pessagno, 1979 \\ Genus Japonocampe Kozur, 1984}

Type species. Triassocampe nova Yao, 1982.

\section{Japonocampe nova (Yao, 1982) Group}

(Pl. 2, fig. 7)

1980 Dictyomitrella sp. B Yao, Matsuda \& Isozaki: pl. 3, figs 1-3. 1982 Triassocampe nova Yao: 59-60, pl. 2, figs 1-4.

1982 Triassocampe nova Yao; Yao, Matsuoka \& Nakatani, pl. 1, fig. 14.

1984 Japonocampe nova (Yao); Kozur: 72.

1986 Triassocampe nova Yao; Bragin: pl. 3, fig. 4.

1986 Triassocampe nova Yao; Yoshida: pl. 4, figs 7, 8.

1991 Triassocampe nova Yao; Bragin: 101, pl. 5, figs 12, 16.

non 1995 Triassocampe nova Yao; Blome \& Reed: 62, pl. 2, fig. 17.

1997 Japonocampe nova (Yao); Sugiyama: 181, fig. 50 (1)

1999 Japonocampe nova (Yao) Group; Tekin: 138, pl. 29, figs 3-5.

Stratigraphic range. Late Triassic; Late Carnian-Middle Norian.

Occurrence. Inuyama Area, Gifu Prefecture and Mino Terrane, Central Japan; Sahalin, Sikhote-Alin, far eastern Russia; Yaylakuzdere and Gökcam sections, Antalya, southwest Turkey.

Family Deflandrecyrtiidae Kozur \& Mostler, 1979

Genus Deflandrecyritium Kozur \& Mostler, 1979

Type species. Deflandrecyritium popofskyi Kozur \& Mostler, 1979.

$$
\text { Deflandrecyrtium parvus Tekin, } 1999
$$

(Pl. 2, fig. 8)

pars 1979 Unnamed Nassellaria Pessagno, Finch \& Abbott: pl. 5 , only fig. 6 .

1982 Squinabolella (?) sp. B Yao: pl. 2, fig. 20.

1996 Unnamed Nassellaria Yeh \& Cheng: pl. 11, figs (?)1, (?)2,

(?)12, 14 and 15.

1997 Haeckelicyrtium sp. A Sugiyama: 156, fig. 41 (9).

1999 Deflandrecyrtium parvus Tekin: 141-142, pl. 30, figs 9-10.

?2000 Haeckelicyrtium sp. A Carter \& Orchard: pl. 2, fig. 12.
Stratigraphic range. Late Triassic; Late Carnian-early Middle Norian-?late Middle Norian.

Occurrence. Baja California Sur, Mexico; Inuyama Area and Mino Terrane, Central Japan; Busuanga Island, Philippines; Yaylakuzdere and Gökcam sections, Antalya, southwest Turkey; ?Queen Charlotte Islands, British Columbia.

Genus Haeckelicyrtium Kozur \& Mostler, 1979 emend. Carter, 1993

Type species. Haeckelicyrtium austriacum Kozur \& Mostler, 1979.

Haeckelicyrtium sp. A

(Pl. 2, figs 9-10)

Description. Cephalis dome shaped, imperforate without horn. Thorax bonnet shaped with small pores. Short abdomen flaring to mainly flat and wide post-abdominal skirt. Three rows of pores situated on post-abdominal skirt. Pore size increasing in width distally.

Stratigraphic range. Late Triassic; late Early Norian-early Middle Norian.

Occurrence. Gökcam section, Antalya, southwest Turkey.

Remarks. Haeckelicyrtium sp. A differs from $H$. subcircularis Tekin by having three rows of pores on abdominal skirt instead of four rows of pores. The former is also differentiated from the latter in having big circular pores on the last row instead of perpendicularly arranged ellipsoidal pores.

Family Syringocapsidae Foreman, 1973 emend. Pessagno, 1977 Genus Syringocapsa Neviani, 1900

Type species. Theosyringium robustum Vinassa, 1901.

Syringocapsa sp. aff. S. batodes De Wever, 1979

(Pl. 2, fig. 12)

aff. 1979 Syringocapsa batodes De Wever in De Wever, San Flippo, Riedel \& Gruber: 292-293, pl. 6, figs 10-12.

Stratigraphic range. Late Triassic; late Early Norian-early Middle Norian.

Occurrence. Gökcam section, Antalya, southwest Turkey.

Remarks. This form differs from Syringocapsa batodes De Wever by having very short proximal (cephalis, thorax and abdomen) part.

Syringocapsa sp. A

(P1. 2, fig. 13)

Description. Test as for genus. Cephalis is dome shaped, imperforate without horn. Thorax and abdomen are sub-cylindrical, imperforate with nodes. Post-abdominal segment inflated with scattered small sub-circular to circular pores. Remnant of tube is at the distal end with small sub-circular pores. 
Stratigraphic range. Late Triassic; late Early Norian-early Middle Norian.

Occurrence. Gökcam section, Antalya, southwest Turkey.

Remarks. Syringocapsa sp. A differs from S. turgida Blome in having less inflated post-abdominal segment with small, scattered pores instead of pentagonal pore frames with large pores.

Family Xiphotecidae Kozur \& Mostler, 1981 Genus Xiphotheca De Wever, 1979

Type species. Xiphotheca karpenissionensis De Wever, 1979.

Xiphotheca longa Kozur \& Mock, 1981 emend. Tekin, 1999 (Pl. 2, fig. 14)

1979 Xiphotheca sp. Pessagno, Finch \& Abbott: pl. 5, fig. 5. 1981 Xiphotheca longa Kozur \& Mock in Kozur \& Mostler: 113-114, pl. 41, fig. 2.

1986 Xiphotheca karpenissionensis De Wever; Sato, Murata \& Yoshida: fig. 16 (14).

1989 Xiphotheca longa Kozur \& Mock; Yeh: 71, pl. 8, fig. 1. 1992 Xiphotheca karpenissionensis De Wever; Otsuka, Kajima \&

Hori: pl. 3, figs 17-18.

1992 Xiphotheca cf. longa Kozur \& Mock; Otsuka, Kajima \& Hori: pl. 3, fig. 19.

1999 Xiphotheca longa Kozur \& Mock; Bragin \& Krylov: 567, figs. $13(\mathrm{E}-\mathrm{G})$.

emend. 1999 Xiphotheca longa Kozur \& Mock; Tekin: 174, pl. 42, figs 13-14.

Stratigraphic range. Late Triassic; latest Carnian/earliest Norian-early Middle Norian-?late Middle Norian.

Occurrence. Baja California, Mexico; West Karpats; Oman; East-Central Oregon, USA; Kyushu, Japan; Yaylakuzdere and Gökcam sections, Antalya, southwest Turkey.

Xiphotheca pseudolonga Tekin, 1999

(Pl. 2, fig. 15)

1999 Xiphotheca pseudolonga Tekin: 174-175, pl. 42, figs 8-11.

Stratigraphic range. Late Triassic; Early Norian-early Middle Norian.

Occurrence. Yaylakuzdere and Gökcam sections, Antalya, southwest Turkey.

\section{Nassellaria Incertae Sedis Genus Canesium Blome, 1984}

Type species. Canesium lentum Blome, 1984.

Canesium lentum Blome, 1984

(Pl. 2, fig. 16)

1979 Eucyritidium (?)sp. A Nakaseko \& Nishimura: 78, pl. 9, figs $5,9$.

1982 Eucyritidium (?)sp. A Yao: pl. 2, figs 9-10.
1982 Eucyritidium (?)sp. A Yao, Matsuoka \& Nakatani: pl. 1, fig. 17.

1984 Canesium lentum Blome: 53-54, pl. 14, figs 3, 8, 11; pl. 17, figs 13 .

1986 Canesium lentum Blome; Yoshida: pl. 6, figs 1, 2.

1999 Canesium lentum Blome; Tekin: 177, pl. 43, fig. 12.

2000 Canesium lentum Blome; Carter \& Orchard: pl. 2, fig. 6

Stratigraphic range. Late Triassic; Late Carnian-early Middle Norian-?late Middle Norian.

Occurrence. Inuyama Area and Gifu Prefecture, Central Japan; East-Central Oregon, USA; Yaylakuzdere and Gökcam sections, Antalya, southwest Turkey; Queen Charlotte Islands, British Columbia.

Genus Enoplocampe Sugiyama, 1997

Type species. Enoplocampe yehae Sugiyama, 1997.

Enoplocampe (?) norica Tekin n. sp.

(Pl. 2, figs 17-18)

Derivation of name. This species is named after its appearance in the Norian.

Diagnosis. Cylindrical form with one or two post-abdominal segments. All segments imperforate with small nodes. Cephalis, hemispherical to dome shaped, with or without a very rudimentary horn. The remainder of the segments are barrel shaped. Thorax with three short to moderately long tricarinate arms.

Holotype. From the sample 00UKT130, illustrated in Plate 2, figure 17.

Material. Six specimens.

Type locality. Gökcam section, Antalya, southwest Turkey (see locality descriptions).

Horizon. Upper Triassic; upper Lower Norian-lower Middle Norian.

Description. Test mainly cylindrical in outline with one or two post-abdominal segments. All segments of the form are imperforate with small nodes. Cephalis, hemispherical to dome shaped, with or without a very rudimentary horn. Thorax barrel shaped with three, short to moderately long triradiate arms with wide grooves and thin ridges, curved inside. Both collar and lumbar strictures marked by shallow depressions. Abdomen barrel shaped, sometimes more inflated than the other segments. The shape of the post-abdominal segments are the same as the thorax and abdomen.

Dimensions $(\mu \mathrm{m})$. Based on four specimens.

$\begin{array}{lrcrr} & \text { HT } & \text { Min. } & \text { Max. } & \text { Av. } \\ \text { Total length of the form } & 183 & 172 & 183 & 178 \\ \text { Max. width of the form } & 96 & 78 & 96 & 86\end{array}$




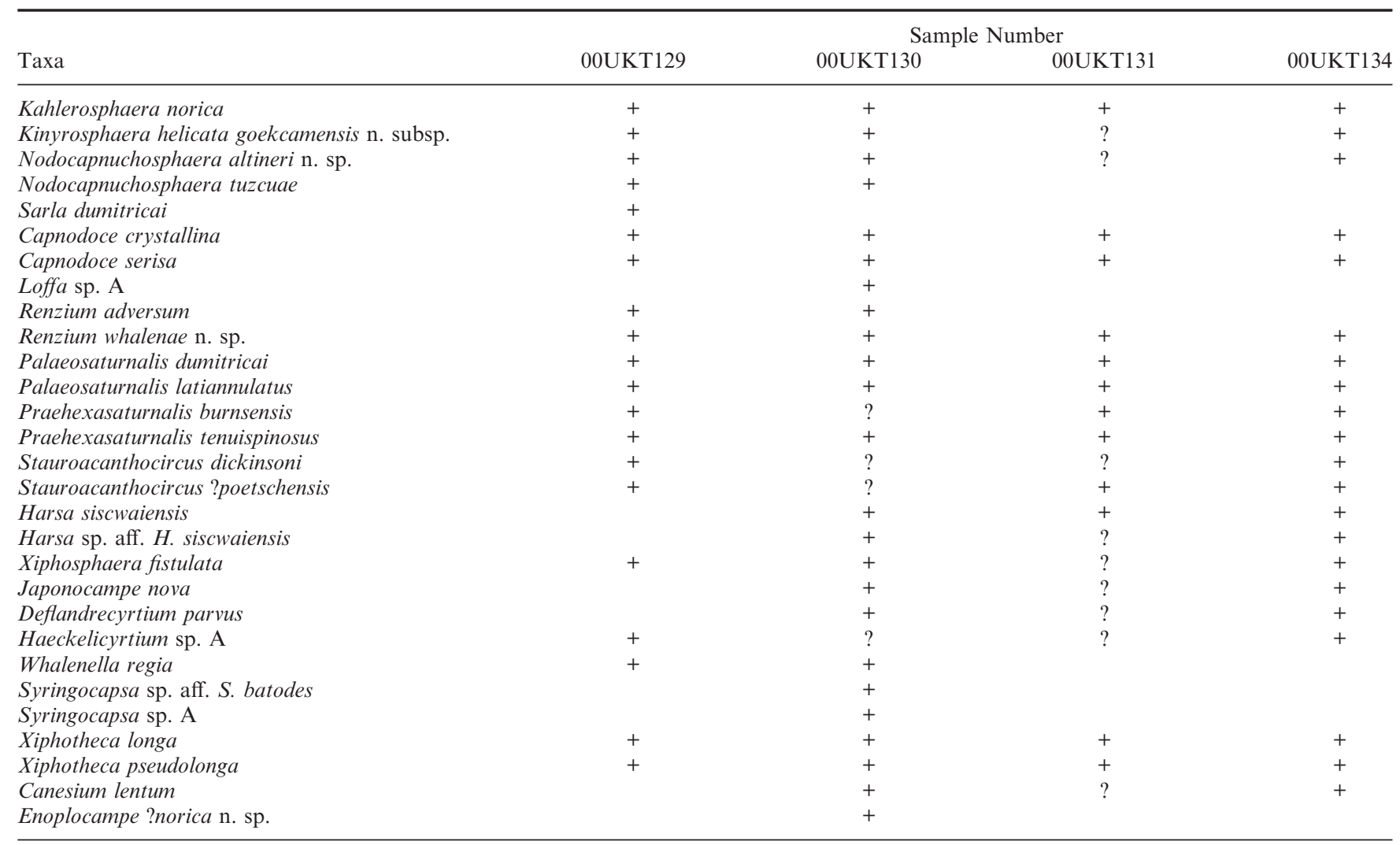

Table 1. Distribution of the late Early to early Middle Norian radiolarians from the Gökdere Formation of the Gökçam section

Stratigraphic range. Late Triassic; late Early Norian-early Middle Norian.

Occurrence. Gökcam section, Antalya, southwest Turkey.

Remarks. Enoplocampe (?) noricaTekin n. sp. is differentiated from $E$. yehae Sugiyama in having a cephalis without a horn or a rudimentary horn, and shorter and wider arms extending out from thorax. This form is tentatively assigned to Enoplocampe Sugiyama due to absence or presence of a very rudimentary apical horn.

Genus Whalenella Kozur, 1984

Type species. Dictyomitra arrecta Hinde, 1908.

Whalenella regia (Blome, 1984)

(Pl. 2, fig. 11)

1984 Corum regium Blome: 51, pl. 13, figs 3, 8, 15.

1986 Corum regium Blome; Yoshida: pl. 5, fig. 4.

1993 Corum regium Blome; Fujii, Hattori \& Nakajima: pl. 3,

fig. 7.

1994 Corum regium Blome; Aita \& Spörli: pl. 7, fig. 5.

1995 Whalenella $\mathrm{cf}$. regia (Blome); Halemic \& Gorican: pl. 1,

figs 21-22.

?1997 Corum regium Blome; Sugiyama: 176, fig. 49 (4).

1999 Corum regium Blome; Tekin: 153, pl. 35, figs 10-11.
Stratigraphic range. Late Triassic; latest Carnian/earliest Norian-early Middle Norian-?late Middle Norian.

Occurrence. East-Central Oregon, USA; Gifu Prefecture and (?) Mino Terrane, Central Japan; Yaylakuzdere and Gökcam sections, Antalya, southwest Turkey.

\section{DATING AND COMPARISION OF THE RADIOLARIAN}

FAUNA

A rich radiolarian fauna has been recovered from the Gökdere Formation of the Gökcam section. Twenty-nine radiolarian taxa have been determined within this fauna (Table 1). Radiolarians obtained from the section are comparable to faunas from the Queen Charlotte Islands, British Columbia (Carter, 1991) and the Yaylakuzdere section, Kemer, Antalya, southwest Turkey (Tekin, 1999).

Within the fauna, Capnodoce serisa, Harsa siscwaiensis, Xiphosphaera fistulata and Saturnalids are very common. According to Carter (1991), Sugiyama (1997) and Tekin (1999), Xiphosphaera fistulata is restricted to the late Early to early Middle Norian in central Japan, the Queen Charlotte Islands (British Columbia) and southwest Turkey. Both Harsa siscwaiensis and $H$. sp. aff. $H$. siscwaiensis are only known from the upper Lower to lower Middle Norian strata of the Queen Charlotte Islands (British Columbia). This is similar to Xiphosphaera fistulata (Carter, 1991). 
According to Carter (1991), although Capnodoce fragilis (junior synonym of $C$. serisa) has a range from the Early Norian (E. abneptis Conodont Zone) to the Middle Norian; its acme occurs in the late Early Norian (E. triangularis Conodont Zone) in Queen Charlotte Islands (British Columbia). Similarly, Tekin (1999) has observed that $C$. serisa first occurs at the top of $E$. abneptis Zone, and then becomes abundant in the E. triangularis Conodont Zone.

Saturnalids such as Praehexasaturnalis burnsensis, P. tenuispinosus and Stauroacanthocircus ?poetschensis are very abundant within the fauna from Gökdere Formation in Gökcam section. Both Praehexasaturnalis burnsensis and $P$. tenuispinosus first appear in the late Early Norian (E. triangularis Conodont Zone) (Tekin, 1999). According to Tekin (1999), Stauroacanthocircus ?poetschensis first appears in the upper part of the E. abneptis Conodont Zone, and becomes abundant in the E. triangularis Conodont Zone in the southwest Turkey. The common occurrence of Paleosaturnalis lupheri, P. macoyoensis (junior synonym of Praehexasaturnalis burnsensis) and $P$. vigrassi (junior synonym of Praehexasaturnalis tenuispinosus) is also reported from the upper Lower Norian strata of the Queen Charlotte Islands, British Columbia (Carter, 1991).

Based on these data, the age of the fauna obtained from the Gökdere Formation of the Gökcam section can be assigned a late Early Norian to early Middle Norian age and the radiolarian association of 'Capnodoce serisa (senior synonym of C. fragilis)-Harsa siscwaiensis-Xiphosphaera fistulata' suggested by Carter (1991) for upper Lower Norian to lower Middle Norian strata can be adopted in this study.

\section{CONCLUSIONS}

The Gökdere Formation in the Alakircay Nappe of the Antalya Nappes is represented by limestone and cherty limestone with chert bands and calciturbidite intercalations. Moderately to well-preserved radiolarians were obtained from the limestone horizons in the Gökdere Formation of the Gökcam section, to the west of Antalya City, southwest Turkey.

Radiolarians obtained from the section are correlated with faunas from the Queen Charlotte Islands, British Columbia (Carter, 1991) and the Yaylakuzdere section, Kemer, Antalya, southwest Turkey (Tekin, 1999). The co-occurrence of Capnodoce serisa, Harsa siscwaiensis, Xiphosphaera fistulata and saturnalids, such as Praehexasaturnalis burnsensis, $P$. tenuispinosus, Stauroacanthocircus ?poetschensis, clearly indicates a late Early Norian to early Middle Norian age for this part of the Gökdere Formation.

Within the radiolarian fauna, four taxa have been determined as new (Kinyrosphaera helicata goekcamensis, Nodocapnuchosphaera altineri, Renzium whalenae, Enoplocampe (?) norica).

\section{ACKNOWLEDGEMENTS}

The authors wish to thank Dr Mustafa Senel for his kind contributions during the preparation of this paper. Scanning electron microscopy for this study was carried out at Innsbruck University, Innsbruck, Austria. Grateful acknowledgements are expressed to Prof. Dr Helfried Mostler for his kind permission to use this facility. We also thank the two reviewers for their assistance.

\section{Manuscript received 23 May 2002 Manuscript accepted 30 June 2003}

\section{REFERENCES}

Aita, Y. \& Spörli, K.B. 1994. Late Triassic Radiolarians from the Torlesse Terrane, Rimutaka Range, Southern North Island, New Zealand. New Zealand Journal of Geology and Geophysics, 37: $155-162$.

Blome, C.D. 1983. Upper Triassic Capnuchosphaeridae and Capnodocinae (radiolaria) from east central Oregon. Micropaleontology, 29(1): $11-49$.

Blome, C.D. 1984. Upper Triassic Radiolaria and radiolarian zonation from western North America. Bulletin of American Paleontology, 85(318): 1-88.

Blome, C.D. \& Reed, K.M. 1995. Radiolarian biostratigraphy of the Quinn River Formation, Block Rock Terrane, north-central Nevada. Correlations with eastern Klamath terrane geology. Micropaleontology, 41(1): 49-68.

Bragin, N.Yu 1986. Triassic biostratigraphy of deposits in South Sahalin. New Proceedings, Academy of Science of the USSR, Moscow, Geological Series. (in Russian), 4: 61-75.

Bragin, N.Yu 1991. Radiolaria and Lower Mesozoic Units of the USSR, east regions. Transactions of the Academy of Sciences of the USSR. (in Russian with English summary), 469: 1-125.

Bragin, N.J. \& Krylov, K.A. 1999. Early Norian Radiolaria from Cyprus. Geodiversitas, 21(4): 539-569.

Brunn, J.H., Dumont, J.F. \& Graciansky, P.C. et al. 1971. Outline of the geology of the western Taurids. In: Campbell, A.S. (Ed.), Geology and History of Turkey. Petroleum Exploration Society of Libya, Tripoli, 225-255.

Carter, E.S. 1991. Late Triassic Radiolarian biostratigraphy of the Kunga Group, Queen Charlotte Islands, British Columbia. In: Woodsworth, G.J. (Ed.), Evolution and Hydrocarbon potential of the Queen Charlotte Basin, British Columbia, 90-10. Geological Survey of Canada, 195-201.

Carter, E.S. 1993. Biochronology and Paleontology of uppermost Triassic (Rhaetian) radiolarians, Queen Charlotte Islands, British Columbia, Canada. Thèse de Doctorat, Université de Lausanne Faculté des Sciences, Mémoires de Géologie (Lausanne), 11: 1-177.

Carter, E.S. \& Orchard, M.J. 2000. Intercalibrated conodontradiolarian biostarigraphy and potential datums for the CarnianNorian boundary within the Upper Triassic Peril Formation, Queen Charlotte Islands, British Columbia. Geological Survey of Canada, Current Research, 2000-A-7: 1-11.

Carter, E.S., Orchard, M.J. \& Tozer, E.T. 1989. Integrated ammonoidcondont-radiolarian biostratigraphy, Late Triassic Kunga Group, Queen Charlotte Islands, British Columbia. Current Research, Part $H$. Geological Survey of Canada Paper, 89-1F: 23-30.

Carter, E.S., Whalen, P.A. \& Guex, J. 1998. Biochronology and paleontology of Lower Jurassic (Hettangian and Sinemurian) radiolarians, Queen Charlotte Islands, British Columbia. Geological Survey of Canada Bulletin, 496: 1-162.

Cordey, F. 1998. Radiolaires des complexes d'accrétion de la Cordillere Canadienne (Colombie-Britannique). Commision Géologique $d u$ Canada Bulletin, 509: 1-209.

De Wever, P. 1981. Hagiastridae, Patulibracchiidae et Spongodiscidae (Radiolaires Polycystines) du Lias de Turquie. Revue de Micropaléontologie, 24(1): 27-50.

De Wever, P. 1982. Radiolaires du Trias et du Lias de la Tethys (Systématique, Stratigraphie). Société Géologique du Nord, Publication, 7(1-2): 1-599.

De Wever, P., Sanflippo, A., Riedel, W.R. \& Gruber, B. 1979. Triassic Radiolaria from Greece, Sicily and Turkey. Micropaleontology, 25(1): $75-110$.

Donofrio, D.A. \& Mostler, H. 1978. Zur Verbreitung der Saturnalidae (Radiolaria) im Mesozoikum der Nördlischen Kalkalpen and Südalpen. Geologisch- Paläontologische Mitteilungen Innsbruck, 7(5): $1-55$. 
Ehrenberg, C.G. 1838. Uber die Bildung der Kreidefelsen und des Kreidemergels durch unsichtbare Organismen. Königliche Preussischen Akademie der Wissenschaften zu Berlin, Abhandlungen, Jahre 1838: $59-147$.

Ehrenberg, C.G. 1875. Fortsetzung der mikrogeologischen Studien als Gesammt Ueberschistder mikroskopischen Palaontologie gleichartig analysirter Gebirgsarten der Erde, mit specieller Rückschit auf den Polysystinen-Mergel von Barbados. Königliche Preussichen Akademie der Wissenschaften zu Berlin, Abhandlungen, Jahre $\mathbf{1 8 8 5}$ $1-225$.

Foreman, H.P. 1973. Radiolaria from DSDP leg 20. In: Heezen, B.C., Mac Gregor, J.D. et al. (Eds), Initial Reports of the Deep Sea Drilling Project, 20. US Government Printing Office, Washington, DC, 249-305.

Fujii, J., Hattori, I. \& Nakajima, T. 1993. A study of radiolarian biostratigraphy and magnetostratigraphy of early Mesozoic red bedded chert, central Japan. News of Osaka Micropaleontologists, Special Publication, 9: 71-89.

Haeckel, E. 1882. Entwurf eines Radiolarien-Systems auf Grund von Studien der Challanger-Radiolarien. Jenaische Zeitschrift für Naturwissenschaft, Jena 15 (n. F. 8): 418-472.

Halemic, J. \& Gorican, S. 1995. Triassic radiolarites from Mts Kalnik and Med Vodnica (Northwestern Croatia). Geologica Croatica, 48(2): 129-146.

Hinde, G.J. 1908. Radiolaria from Triassic and other rocks of the Dutch East Indian Archipelago. Jaarboek net Mijnwezen Nederlansch Oost-Indie, 37: 694-736 (édition française 709-751).

Kalafatcioglu, A. 1973. Antalya korfezi bati kesiminin jeolojisi. Maden Tetkik ve Arama Bülteni, 81: 82-131 (in Turkish).

Kishida, Y. \& Sugano, K. 1982. Radiolarian zonation of Triassic and Jurassic in outer side of Southwest Japan. News of Osaka Micropaleontologists, Special Volume (in Japanese with English abstract), $\mathbf{5}$ 271-300.

Knipper, A.L., Satian, M.A. \& Bragin, N. Yu 1997. Upper TriassicLower Jurassic Volcanogenic and sedimentary deposits of the Old Zos pass (Transcaucasia). Stratigraphy and Geological Correlation, 5(3): $58-65$.

Kozur, H. 1984. New Radiolarian taxa from the Triassic and Jurassic. Geologisch-Paläontologische Mitteilungen Innsbruck, 13(2): 49-88.

Kozur, H. \& Mostler, H. 1972. Beiträge zur Erforschung der mesozoischen Radiolarien. Teil. 1, Revision der Oberfamilie Coccodiscacea HAECKEL, 1862 emend. und Beschreibung ihrer triassischen Vertreter. Geologisch-Paläontologische Mitteilungen Innsbruck, 2(8/9): $1-60$.

Kozur, H. \& Mostler, H. 1979. Beiträge zur Erforschung der mesozoischen Radiolarien.Teil III. Die Oberfamilien Actinommacea HAECKEL, 1862 emend., Artiscacea HAECKEL, 1882, Multiarcusellacea nov. Der Spumellaria und triassische Nassellaria. Geologisch-Paläontologische Mitteilungen Innsbruck, 9(1-2): 1-132.

Kozur, H. \& Mostler, H. 1981. Beiträge zur Erforschung der mesozoischen Radiolarien. Teil IV. Thalossosphaeracea HAECKEL, 1862, Hexastylacea HAECKEL, 1862 emend. Petruhevskaya, 1979, Sponguracea HAECKEL, 1862 emend. und weitere triassische Lithocycliacea, Trematodiscacea, Actinommacea und Nassellaria. Geologisch-Paläontologische Mitteilungen Innsbruck, 1: 1-208.

Kozur, H. \& Mostler, H. 1983. The polyphyletic origin and the classification of the Mesozoic saturnalids (Radiolaria). GeologischPaläontologische Mitteilungen Innsbruck, 13: 1-47.

Kozur, H. \& Mostler, H. 1990. Saturnaliacea Deflandre and some others stratigraphically important Radiolaria from the Hettangian of Lenggries/Isar (Bavaria, Northern Calcareous Alps). GeologischPaläontologische Mitteilungen Innsbruck, 17: 179-248.

Lahm, B. 1984. Spumellarianfauna (Radiolaria) aus dem Mitteltriassichen buchensteiner Schihten von Recoaro (Norditalien) und den Obertriasichen reiflingerkalken von Grossreifling (Osterrich)Systematik-Stratigraphie. Münchener Geowissenschaftliche Abhandlungen Part A, 1: 1-161.

Lefevre, R. 1967. Nouvel élément dans la géologie du Taurus Lycien: les Nappes d'Antalya (Turquie). Comptes Rendus de l'Academie des Sciences, Paris, 7(D 265): 1365-1368.

Matsuda, T. \& Isozaki, Y. 1982. Radiolarians around the TriassicJurassic boundary from the bedded chert in the Kamiso Area,
Southwest Japan. News of Osaka Micropaleontologists, Special Volume, 5: 93-102.

Müller, J. 1858. Uber die Thalassicollen, Polycystinen und Acanthometren des Mittelmeeres. Abhandlungen der Preussischen Akademie der Wissenschafteler zu Berlin, Jahrang, 1858: 1-62.

Nakaseko, K. \& Nishimura, A. 1979. Upper Triassic Radiolaria from southwest Japan. Scientific Report of College Education, Osaka University, 28(2): 61-109.

Neviani, A. 1900. Supplemanto alla fauna a radiolari dela rocce Mesozoiche indel Bolognase. Bulletino Societa Geologico Italiana, 19: 645-671.

Nishizono, Y. \& Murata, M. 1983. Preliminary studies on the sedimentary facies and radiolarian biostratigraphy of Paleozoic and Mesozoic sediments, exposed along the mid-stream of the Kuma River, Kyushu, Japan. Kumomoto Journal of Science Geology, 12: 1-40.

Otsuka, T., Kajima, M. \& Hori, R. 1992. The Batinah Olistostrome of the Oman Mountains and Mesozoic Radiolarians. News of Osaka Micropaleontologists, Special Volume, 8: 21-34.

Özgül, N. 1976. Toroslarin bazi temel jeolojik özellikleri. Türkiye Jeoloji Kurumu Bülteni, 19: 65-78 (in Turkish).

Özgül, N. 1984. Stratigraphy and tectonic evolution of the Central Taurides. In: Tekeli, O. \& Göncüoglu, C. (Eds), Geology of the Taurus Belt. Publication of General Directorate of Mineral Research and Exploration, Ankara, 77-90.

Pessagno, E.A. Jr 1977a. Upper Jurassic Radiolaria and radiolarian biostratigraphy of the California Coast Ranges. Micropaleontology, 23(1): $56-113$.

Pessagno, E.A. Jr \& Blome, C.D. 1980. Upper Triassic and Jurassic Pantanelliinae from California, Oregon and British Columbia. Micropaleontology, 26(3): 225-273.

Pessagno, E.A. Jr \& Newport, R.L. 1972. A new technique for extracting Radiolaria from radiolarian cherts. Micropaleontology, 18(2): 231-234.

Pessagno, E.A. Jr, Finch, W. \& Abbott, P.L. 1979. Upper Triassic radiolaria from San Hipolito Formation, Baja California. Micropaleontology, 25(2): 160-197.

Robertson, A.H.F. \& Woodcock, N.A. 1981. Gödene Zone, Antalya Complex, Volcanism and sedimentation along a Mesozoic continental margin, SW Turkey. Geologische Rundaschau, 70(3): 1177-1214.

Rüst, D. 1885. Beiträge zur Kenntniss der Fossilen Radiolarien aus Gesteinen des Jura. Palaeontolographica, 31: 269-321.

Sato, T., Nishizono, Y. \& Murata, M. 1982. On the Jurassic Radiolarian faunas from the Shakumasan Formation. News of Osaka Micropaleontologists, Special Volume, 5: 301-310.

Sato, T., Murata, M. \& Yoshida, H. 1986. Triassic to Jurassic radiolarian biostratigraphy in the southern part of Chichibu terrane of Kyushu, Japan. News of Osaka Micropaleontologists, Special Volume, 7: 9-23.

Senel, M. 1997a. 1/100.000 ölcekli Türkiye Jeoloji Haritalari, Antalya- L 10 paftasi. Maden Tetkik ve Arama Genel Müdürlügü Yayinlari, 7: 1-22 (in Turkish).

Senel, M. 1997b. 1/100.000 ölcekli Türkiye Jeoloji Haritalari, Antalya- L 11 paftasi. Maden Tetkik ve Arama Genel Müdürlügü Yayinlari, 8: $1-15$ (in Turkish).

Senel, M., Kengil, R., Ünverdi, M., Gözler, M.Z. \& Serdaroglu, M. 1981. Teke Toroslari guneydogusunun jeolojisi. Maden Tetkik ve Arama Dergisi, 95/96: 13-43 (in Turkish).

Senel, M., Dalkilic, H. \& Gedik, I. 1992.et al. Egirdir-YenisarbademliGebiz ve Geris-Koprulu (Isparta-Antalya) arasinda kalan alanlarin jeolojisi. Maden Tetkik ve Arama Genel Müdürlügü Report No. 9390: 1-559 (unpublished) (in Turkish).

Sugiyama, K. 1997. Triassic and Lower Jurassic Radiolarian biostratigraphy in the siliceous claystone and bedded chert units of the southeastern Mino Terrane, Central Japan. Bulletin of the Mizunami Fossil Museum, 24: 79-193.

Tekin, U.K. 1999. Biostratigraphy and systematics of late middle to late Triassic radiolarians from the Taurus Mountains and Ankara Region, Turkey. Geologisch-Paläontologische Mitteilungen Innsbruck, 5: $1-297$.

Tekin, U.K. 2002a. Late Triassic (Late Norian-Rhaetian) radiolarians from the Antalya Nappes, Central Taurids, Southern Turkey. Rivista Italiana Paleontologia e Stratigrafia, 108(3): 415-440. 
Tekin, U.K. 2002b. Lower Jurassic (Hettangian-Sinemurian) radiolarians from the Antalya Nappes, Central Taurides, Southern Turkey. Micropaleontology, 48(2): 177-205.

Yang, Q. \& Mizutani, A. 1991. Radiolaria from the Nadanhada Terrane, northeast China. The Journal of Earth Sciences, Nagoya University, 38: 49-78.

Yao, A. 1982. Middle Triassic to Early Jurassic radiolarians from the Inuyama Area, Central Japan. Journal of Geoscience, Osaka City University, 25: 53-70.

Yao, A., Matsuda, T. \& Isozaki, Y. 1980. Triassic and Jurassic radiolarians from the Inuyama area, central Japan. Journal of Geological Science of Osaka, 3(4): 135-154.

Yao, A., Matsuoka, A. \& Nakatani, T. 1982. Triassic and Jurassic radiolarian assemblage in the southmost Japan. News of Osaka
Micropaleontologists, Special Volume. (in Japanese with English abstract), 5: 27-43.

Yeh, K. 1989. Studies of Radiolaria from the Fields Creek Formation, East-Central Oregon, USA. Bulletin of the National Museum of Natural Sciences, Taiwan, 1: 43-110.

Yeh, K. 1990. Taxonomic studies of Triassic Radiolaria from Busuanga Island, Philippines. Bulletin of the National Museum of Natural Sciences, Taiwan, 2: 1-63.

Yeh, K. \& Cheng, Y. 1996. An Upper Triassic (Rhaetian) Radiolarian Assemblage from Busuanga Island, Philippines. Bulletin of the National Museum of Natural Sciences, Taiwan, 7: 1-43.

Yoshida, A. 1986. Upper Triassic to Lower Jurassic radiolarian biostratigraphy in Kagamigahara City, Gifu Prefecture, Central Japan. Journal of Earth Science, Nagoya University, 34: 1-21. 